\title{
Heat stress-induced renal damage in poultry and the protective effects of HSP60 and HSP47
}

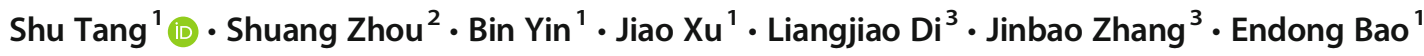 \\ Received: 6 March 2018 / Revised: 1 May 2018 / Accepted: 3 May 2018 / Published online: 20 May 2018 \\ (C) Cell Stress Society International 2018
}

\begin{abstract}
The present study investigates the effects of heat stress on the kidney in broilers, based on previous findings which showed that heat stress caused cardiac damage in broilers. Further, the possible renoprotective role of aspirin and the heat shock proteins HSP60 and HSP47 was also investigated. The enzyme levels of urea and uric acid, which are indicators of renal damage, and lactate dehydrogenase, an indicator of oxidative damage, were measured in chickens that were only exposed to heat stress, chickens that were pretreated with aspirin before heat stress, and chickens that were only treated with aspirin. Further, histological examination of renal tissue from the three groups was also performed. Finally, expression of HSP60 and HSP47 was also examined. In the heat stress group, the enzyme measurements were indicative of renal dysfunction and oxidative damage, and the histological findings were indicative of renal ischemia and damage. Aspirin seemed to have a protective effect against the renal damage caused by the stress, based on the enzyme measurements and histopathological findings in the aspirin-treated group. The findings also indicate that aspirin may induce HSP60 and HSP47 expression in renal cells. Finally, the expression patterns of HSP60 and HSP47 indicated that they may play a renoprotective role, as their expression was higher in the aspirintreated groups. In conclusion, the present findings show that heat stress causes renal damage in poultry and that aspirin may play a protective role against this damage via pathways that involve HSP60 and HSP47.
\end{abstract}

Keywords Heat stress $\cdot$ Poultry $\cdot$ Renal dysfunction $\cdot$ Aspirin $\cdot$ Heat shock proteins

\section{Introduction}

Heat stress can affect animal health and cause damage to several organs and even lead to sudden death. Economic losses resulting from heat stress have been reported worldwide (Allakhverdiev et al. 2008; Barrow and Clark 1998). Poultry are more sensitive to heat stress than mammals because they lack sweat glands. Therefore, during the summer, chickens are more sensitive to heat than other livestock. As a non-specific stressor, heat stress causes damage to multiple organs (Bao et al. 2008). Our previous studies have confirmed that heat stress

Shu Tang

dandan0107@163.com

College of veterinary Medicine, Nanjing Agricultural University, Nanjing 210095, Jiangsu, China

2 College of animal science and technology, Nanjing Agricultural University, Nanjing 210095, Jiangsu, China

3 Zoohance Biotech Co., Ltd, Yinchuan 750001, Ningxia, China in chickens can cause serious harm to the heart. However, we did not pay attention to other organs that were also damaged, especially the kidney. Therefore, in the present study, we focused on renal injury caused by heat stress in broilers.

Heat shock protein (HSP) expression is induced in cells in response to various types of stress. These proteins play an essential role in many physiological cellular processes, such as protein folding, transport, and assembly. According to their molecular weight, HSPs are divided into several families, such as small heat shock protein family, HSP47, HSP60, HSP70, HSP90, and HSP110 (Lin et al. 2004). Our previous research showed that HSPs protect myocardial cells from the effects of heat stress (Bao et al. 2008; Blum and Miller 1998; Tang et al. 2014). Therefore, HSPs may also play an important role in the protection of renal cells in broilers exposed to heat stress. This is another point of investigation in the present study.

Hsp60, also known as HspD1 and chaperonin 60, has been widely recognized as a molecular chaperone responsible for cellular stress response (Bukau and Horwich 1998; Buriro et al. 2012; Kuo et al. 2012). In addition, Hsp60 (HSPD1) is also reported to be involved in the regulation of intracellular 
protein aggregation, ATP production, and oxidative stress in diabetes-induced renal tubular dysfunction (Lebret et al. 2003). The kidney is abundant in mitochondria, which are involved in supplying ATP for several metabolic pathways in renal tubular cells. Hsp60 is well known as a mitochondrial chaperone that can stabilize the inner and outer membrane of the mitochondria and prevent the apoptosis of cells (Bruschi et al. 1993; Reading et al. 1989). Thus, Hsp60 could play a role in the protection of renal cells from heat stress in chickens.

Another Hsp that could be involved in the protection of renal cells from heat stress is Hsp47. Under conditions of stress, Hsp47 is part of the quality control system for procollagen, including the prevention of the secretion of procollagen with abnormal conformation (Hamilton and Heikkila 2006; Nagata and Hosokawa 1996). In addition to its role as a molecular chaperone, Hsp47 synthesis always parallels that of collagen in developing tissues and various cell lines, and in collagen-related pathological conditions such as fibrosis (Razzaque and Taguchi 1999). Moriyama reported that cells expressing Hsp47 are presumably myofibroblasts, which appear during the progression of interstitial fibrosis in the rat kidney and play a protective role in kidney cells (Razzaque and Taguchi 1999; Xiao et al. 2012). Therefore, in addition to Hsp60, Hsp47 may also be involved in renoprotection in chickens exposed to heat stress.

Aspirin is a medication that is often used to treat pain, fever, and inflammation (WM Murray 1997). Aspirin exerts its effects by mediating, at least in part, the inhibition of cyclooxygenase (COX) enzymes and reduced production of prostaglandins and other inflammatory mediators. Heat stress can activate multiple body response pathways that involve inflammation. Our previous studies have confirmed that aspirin could protect the heart and induce the expression of several Hsp's in broilers under heat stress. In addition, aspirin irreversibly inhibits the production of thromboxane and hence prevents platelet aggregation. Its role is well established in the secondary prevention of cardiovascular disease (CVD), and its role in the primary prevention of CVD in chronic kidney disease (CKD) has been identified as an important research priority (Evans et al. 2009; Wali and Wali 2010). In the present study, we fed broilers aspirin $(1 \mathrm{mg} / \mathrm{kg}) 2 \mathrm{~h}$ before exposure to heat stress; this dose is much lower than the average dose given and is therefore safe. Our previous studies have confirmed that aspirin could induce the expression of several Hsp's such as Hsp70, Hsp90, and Hsp27 and CryAB. Inhibition of these Hsp's by a specific inhibitor could weaken aspirin's protective role in the chicken heart under in vitro heat stress conditions. Next, we sought to investigate renal damage in the chicken under heat stress and whether aspirin could also have a protective effect in renal tissue.

In this study, we investigated the effects of heat stress on renal cells in broiler chickens. Further, the expression of Hsp60 and Hsp47 in the renal cells was also investigated.
We also studied their expression in chickens that were treated with aspirin (acetyl salicylic acid), since our previous studies indicated that aspirin could protect chicken primary myocardial cells under heat stress both in in vivo and in vitro conditions (Wu et al. 2015).

\section{Materials and methods}

\section{Establishment of the in vivo heat stress model}

All experiments were performed in accordance with the guidelines of the Animal Ethics Committee of Jiangsu Province (China) and were approved by the Institutional Animal Care and Use Committee of Nanjing Agricultural University, China. This research was conducted in accordance with the guidelines of the Institutional Animal Care and Use Committee (IACUC), as is mandatory for all submissions with research involving live animals. The in vivo heat stress model was established as previously described (Wu et al. 2015). Briefly, 1-day-old specific pathogen-free chickens were purchased from Qian Yuan Hao Biotechnology Company, Nanjing, China. The entire population was vaccinated against Newcastle disease and infectious bursal disease on day 7 and day 14 , respectively. The birds were allowed to acclimate to their new housing and recover from environmental stress for 30 days. Then, 270 chickens were randomly divided into three groups: the Heat (the chickens were only exposed to heat stress), the Aspirin + Heat group (the chickens were pretreated with aspirin before heat stress), and the Aspirin group (the chickens were pretreated with aspirin, but not exposed to heat stress) ( $n=90$ in each group). The chickens were not given water in the $12 \mathrm{~h}$ leading up to the experiment. According to our observation, broilers did not present dehydration before the experiment. We did pre experiment and found when to stop giving water for $12 \mathrm{~h}$ (about one night) for chicks. Chickens can drink all aspirin added water in $2 \mathrm{~h}$ before heat treatment. The chickens in the Aspirin and Aspirin + Heat groups were administered aspirin orally at $1 \mathrm{mg} / \mathrm{kg}$ body weight (aspirin powder $>98 \%$ purity; Sigma, USA) $2 \mathrm{~h}$ before the heat stress phase of the experiment. During the heat stress phase, the chickens in the Aspirin group were housed under normal conditions (i.e., they were not exposed to heat), while the chickens in the Aspirin + Heat and Heat groups were exposed to heat stress by rapidly, gently moving the animals from the $25 \pm 1{ }^{\circ} \mathrm{C}$ housing to an air chamber (GJ-1; Suzhou Fengshi Laboratory Animal Equipment Co. Ltd., China) preheated to $40 \pm 1{ }^{\circ} \mathrm{C}$ at $60-70 \%$ humidity. The broilers had ad libitum access to food and water during heat stress exposure. At 0 (control), 1, 2, 3, 5, 7, 10, 15, and $24 \mathrm{~h}$ after heat stress exposure, at the end of each time point, 10 chickens were randomly selected and sacrificed humanely by rapid decapitation (within $5 \mathrm{~min}$ ). The body temperature of the broilers 
was measured via the rectum (with a thermometer placed $3 \mathrm{~cm}$ inside) using a mercury thermometer within 2 min before slaughter. The kidneys were excised and fixed in $10 \%$ formalin for pathological studies or frozen in liquid nitrogen for ELISA.

\section{Detection of enzymes associated with renal damage}

Serum $(1.5 \mathrm{~mL})$ was collected at every time point $(0,1,2,3,5$, $7,10,15$, and $24 \mathrm{~h}$ ) from the chickens within $3 \mathrm{~min}$ after exposure to the treatments described above. The lactate dehydrogenase (LDH), urea, and uric acid (UA) activity was measured according to the manufacturer's instructions (Nanjing Jiancheng Biochemical Reagent Co. Ltd., Nanjing, China) with a clinical biochemical indicator auto-analyzer (Vital Scientific NV, The Netherlands). Each sample was analyzed three consecutive times.

\section{Detection of HSP60 and HSP47 expression}

The chicken kidney samples were washed in ice-cold saline and homogenized on ice in 10 volumes of homogenization buffer $(0.15 \mathrm{M} \mathrm{NaCl}, 20 \mathrm{mM}$ Tris- $\mathrm{HCl}$ [pH 8.0], $1 \mathrm{mM}$ ethylenediaminetetraacetic acid, $1 \mathrm{mM}$ phenylmethylsulphonyl fluoride, $0.1 \mu \mathrm{M}$ E-46, $0.08 \mu \mathrm{M}$ aprotinin, $0.1 \mu \mathrm{M}$ leupeptin, and $0.1 \%$ NP-40) using an Ultra-Turrax homogenizer (623,003; Fluko Equipment Shanghai Co. Ltd., China). The homogenates were centrifuged at $12,000 \times g$ for $20 \mathrm{~min}$ at $4{ }^{\circ} \mathrm{C}$ to remove debris, and the supernatant was collected and stored at $-20{ }^{\circ} \mathrm{C}$ for protein quantification. The protein concentration of the samples was measured using a Micro-BCA ${ }^{\mathrm{TM}}$ protein assay kit $(23,235$; Thermo Scientific, Waltham, MA, USA), and the HSP47 and HSP60 protein levels were quantified using a commercially available ELISA kit (for Hsp47: MBS036095 [detection range in chicken, 0.1-40 ng/mL]; for Hsp60: MBS9310299 [detection range in chicken, 0.1 $-40 \mathrm{ng} / \mathrm{mL}]$ ) (MyBioSource, USA) according to the manufacturer's instructions.

\section{Histopathological analysis}

Kidney tissue samples were obtained and preserved in $10 \%$ formalin. The samples were embedded in paraffin and then cut into serial sections of $5 \mu \mathrm{m}$ thickness. Sections were stained with $H \& E$, and images were obtained with a light microscope (Imager A2; ZEISS, Germany).

\section{Statistical analysis}

The Curve Expert 1.3 software was used to generate the standard curves for the ELISA results. Data were compared with the baseline level ( $0 \mathrm{~h}$ in the HS group) by one-way analysis of variance (ANOVA) followed by Fisher's least significant difference (LSD) test using SPSS version 21 for Windows. The three groups (Heat, Aspirin + Heat, and Aspirin) were compared using two-way ANOVA with the Graphpad prism 6.0 software. When $p$ values were $<0.05$ ("significant difference vs. the 0 -h heat stress group, " significant difference vs. the Heat group, and significant symbol was polylines between Aspirin + Heat and Aspirin group), the differences were considered to be significant, and when they were $<0.01\left(^{* *}\right.$, \#\#), the differences were considered to be highly significant. Duncan's multiple range test was used to compare Hsp47 and Hsp60 expression between groups at each time point. All raw data presented are expressed as the mean \pm standard deviation (SD) values. All experiments were performed in triplicate.

\section{Results}

\section{Clinical manifestations}

The normal body temperature in the chicken is $39^{\circ} \mathrm{C}$, and all the control chickens (no heat stress and only Aspirin groups) showed this temperature. The range of a mercury thermometer is from 25 to $43^{\circ} \mathrm{C}$. The body temperature of most broilers in the Heat group was over $43^{\circ} \mathrm{C}$. We assumed that it was $44^{\circ} \mathrm{C}$ since we were unable to measure the temperature if it was above $43{ }^{\circ} \mathrm{C}$. Broilers did not show deviation from their normal body temperature when they were fed aspirin with water $2 \mathrm{~h}$ before heat treatment. At $1 \mathrm{~h}$ after heat treatment, broilers in the Heat groups presented with polypnea and sensitive compared with the Aspirin + Heat pretreated group. At $5 \mathrm{~h}$ after heat stress, the broilers in the Heat group were not as active as those in the Aspirin treatment group. The Aspirin + Heat treatment group presented with similar behavior, and the body temperature remains between 41 and $42^{\circ} \mathrm{C}$. At $10-24 \mathrm{~h}$ after heat stress, broilers pretreated with aspirin in the Aspirin + Heat groups showed relaxed behavior in the chamber. According to our rectal temperature data, both treatment groups (Heat and Aspirin + Heat) showed higher rectal temperature than the control group $\left(25^{\circ} \mathrm{C}\right)$. However, in the Heat stress groups, nearly half of the broilers showed higher temperature (over $43{ }^{\circ} \mathrm{C}$ ) than the Aspirin + Heat group (41 $\left.-43^{\circ} \mathrm{C}\right)$.

\section{Serum LDH levels}

The non-heat treatment Aspirin and control groups showed similar serum LDH values (control, $955 \mathrm{U} / \mathrm{L}$; Aspirin, $1053 \mathrm{U} / \mathrm{L}$ ). After $1 \mathrm{~h}$ of heat stress, serum LDH levels in the Heat group increased by 2.66 -fold ( $2525 \mathrm{U} / \mathrm{L}$ ) compared with the Aspirin (948 U/L) group. Compared to the aspirin treatment groups (with heat and without heat), the LDH level in the heat treatment group remained significantly higher from 1 to $24 \mathrm{~h}(>2000 \mathrm{U} / \mathrm{L})$. From 3 to $7 \mathrm{~h}$, the LDH levels in the 
Aspirin + Heat group $(3 \mathrm{~h}, 1044 \mathrm{U} / \mathrm{L} ; 5 \mathrm{~h}, 2225 \mathrm{U} / \mathrm{L} ; 7 \mathrm{~h}$, $1789 \mathrm{U} / \mathrm{L})$ were higher than those in the Aspirin group $(p<$ $0.01)$, but the LDH levels were similar between these two groups at all other time points $(\sim 1000 \mathrm{U} / \mathrm{L})$. The LDH level in the group only treated with aspirin did not fluctuate much $(p>0.05)$ (Fig. 1).

\section{Serum urea and UA levels}

The serum urea level in the Heat group significantly increased from 2 to $24 \mathrm{~h}(>1,1.34,1.52,1.44$, and $1.33 \mathrm{U} / \mathrm{L}$ at $2,7,10$, and 24 h, respectively) (Fig. 2a). In the Aspirin + Heat group, the urea level increased slightly from $2 \mathrm{~h}(0.85 \mathrm{U} / \mathrm{L})$ under heat stress, but it was much lower $(<1 \mathrm{U} / \mathrm{L})$ than that in the Heat group $(p<0.01)$ over the 24 -h duration. In the Aspirin group, the urea levels did not fluctuate much throughout the 24-h duration $(<0.5 \mathrm{U} / \mathrm{L})$. The UA levels showed similar trends to the urea levels (Fig. 2b).

\section{Histopathological features of the kidney}

As can be seen in Fig. $3 g-i$, heat stress resulted in severe damage in renal tubule epithelial cells: at $5 \mathrm{~h}$, karyopyknosis ( $\Delta$ ) and acute degeneration $(\uparrow)$ can be observed; at $24 \mathrm{~h}$, severe cell death was the main pathological change in renal tubule epithelial cells, characterized by disappearance of the nucleus and karyopyknosis $(\boldsymbol{\Delta})$. In the Aspirin + Heat group (Fig. $3 \mathrm{~d}-\mathrm{f}$ ), after $5 \mathrm{~h}$ of heat treatment, the renal tubule epithelial cells showed acute degeneration $(\uparrow)$, and after $24 \mathrm{~h}$ of heat stress, acute degeneration was still the main pathological change observed. Karyopyknosis can be observed, but few lesions were present in comparison to the Heat stress group. In the Aspirin group (Fig. $3 \mathrm{a}-\mathrm{c}$ ), no obvious damage to renal tubule epithelial cells can be seen.

\section{Hsp47 and Hsp60 expression in the kidney}

As shown in Fig. 4a, in the Heat stress group, the HSP47 level increased at 2 and $4 \mathrm{~h}$ (0.58 and $0.51 \mathrm{ng} / \mathrm{mL}$, respectively) and then decreased from 5 to $24 \mathrm{~h}$ (to $\sim 0.45 \mathrm{ng} / \mathrm{mL}$ ). The group that was only treated with aspirin showed an increase in HSP47 levels from 5 to $10 \mathrm{~h}(\sim 0.6 \mathrm{ng} / \mathrm{mL})$, but they were lower than the levels in the heat stress group at 15 to $24 \mathrm{~h}(0.31$ and $0.35 \mathrm{ng} / \mathrm{mL}$, respectively). In the aspirin treatment group, after heat stress was induced, the Hsp47 levels were significantly higher (average, $>0.55 \mathrm{ng} / \mathrm{mL}$; from 5 to $24 \mathrm{~h},>$ $0.60 \mathrm{ng} / \mathrm{mL}$ ) than those in the only heat treatment group throughout the treatment time. The group that was only treated with aspirin showed decreased levels of Hsp47 from $15 \mathrm{~h}$, which is consistent with the half-life of aspirin.

As shown in Fig. 4b, Hsp60 expression in the Heat group $(0.73 \mathrm{ng} / \mathrm{mL})$ was lower than that in Aspirin + Heat and Aspirin groups $(0.97 \mathrm{ng} / \mathrm{mL})$ at $0 \mathrm{~h}$. After heat stress for $1 \mathrm{~h}$,

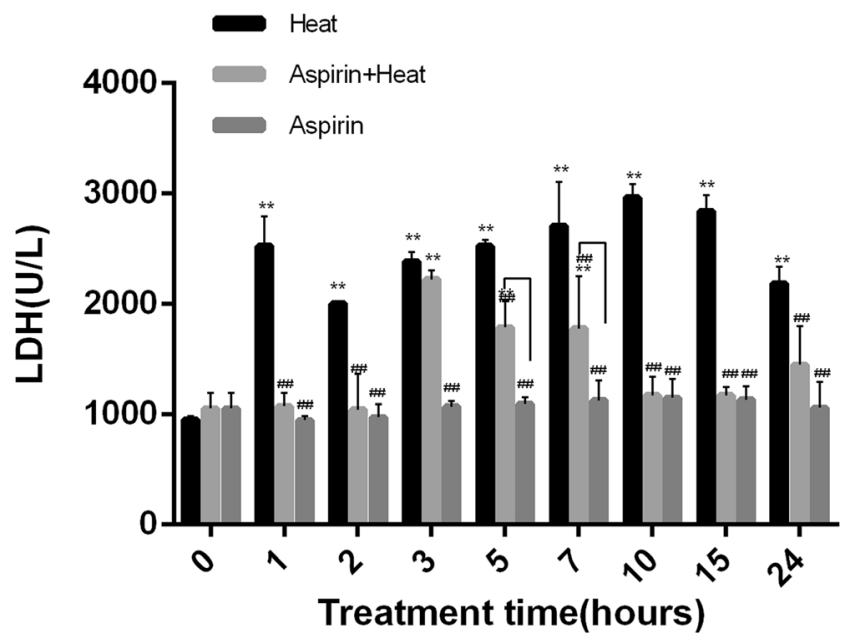

Fig. 1 Changes in LDH levels in response to heat stress. Compared to the Aspirin group, in the Heat group, the LDH level was significantly higher from 1 to $24 \mathrm{~h}\left({ }^{* *} p<0.01\right)$. From $3 \mathrm{~h}$, the Aspirin + Heat group showed higher LDH levels than that at $0-2 \mathrm{~h}$ of heat stress, but they were still lower than those in the Heat group. From $10 \mathrm{~h}$, the LDH level decreased until $24 \mathrm{~h}$ compared to the Heat group $\left({ }^{\# \#} p<0.01\right)$. The Aspirin group showed similar LDH levels throughout. Lines indicate significant differences between the Aspirin + Heat and Aspirin groups

the Hsp60 level in the Heat group $(1.05 \mathrm{ng} / \mathrm{mL})$ was higher than that in the other two groups $(0.65 \mathrm{ng} / \mathrm{mL}$ in the Aspirin + Heat group and $0.66 \mathrm{ng} / \mathrm{mL}$ in the Aspirin group) until $7 \mathrm{~h}$, but after $10 \mathrm{~h}$, Hsp60 expression was lower $(<0.65 \mathrm{ng} / \mathrm{mL})$ than that in the other two groups $(>0.65 \mathrm{ng} / \mathrm{mL})$. The aspirintreated groups showed higher Hsp60 expression than the Heat group before heat stress, but it decreased after heat stress in the Aspirin + Heat group until $10 \mathrm{~h}(p<0.05)$, after which it increased again after $15 \mathrm{~h}$ but remained similar to the level in the Aspirin group. The aspirin treatment group without heat stress showed similar trends with the Aspirin + Heat group until $10 \mathrm{~h}$, but from $15 \mathrm{~h}$, Hsp60 expression was lower than that in the Aspirin + Heat group.

\section{Correlation analysis}

The Pearson correlation coefficients for the correlation between kidney damage indicators and Hsp expression in the broiler kidney are presented in Table 1. The Pearson correlation coefficient for the relationship between UA and Hsp47 was 0.377 , which indicated a significant positive correlation. The coefficient for the correlation between UA and Hsp60 was -0.125 ; between urea and Hsp47, -0.411 ; between urea and Hsp60, -0.403 . These values indicated a significant negative correlation.

\section{Discussion}

Heat stress is kind of non-specific stressor that could affect the welfare of livestock and even result in death. In the present 


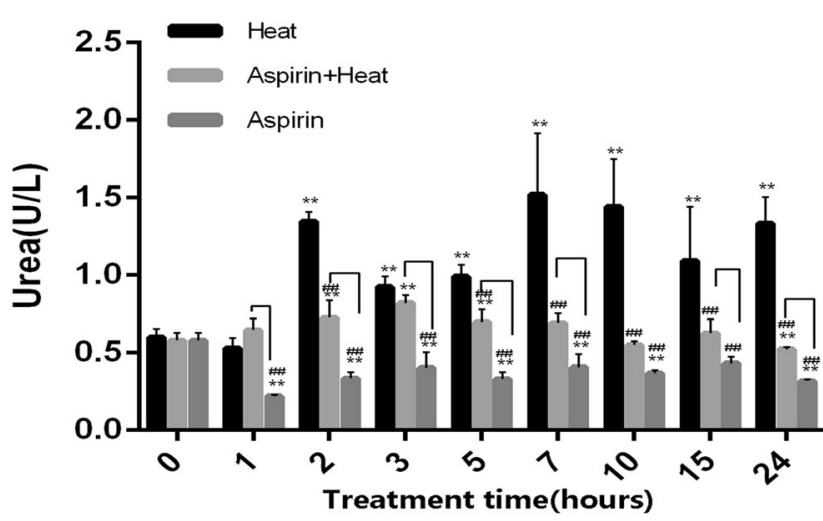

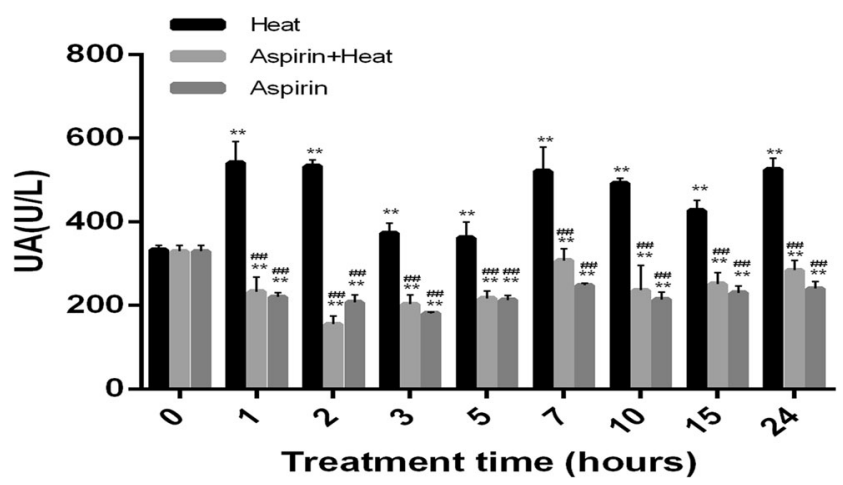

Fig. 2 Changes in urea and UA levels in response to heat stress. a After $2 \mathrm{~h}$ of heat stress, the urea levels were significantly higher in the Heat group than in the Aspirin group $\left({ }^{* *} p<0.01,{ }^{\# \#} p<0.01\right)$. The Aspirin + Heat group had lower urea levels than the Heat group ( $\left.{ }^{\#} p<0.01\right)$. The Aspirin group had lower urea levels than the Heat group at all time points $\left({ }^{\# \#} p<0.01\right)$. Lines indicate significant differences between the Aspirin + Heat and Aspirin groups. b After $1 \mathrm{~h}$ of heat stress, the UA levels were significantly higher in the Heat group than in the Aspirin group until $24 \mathrm{~h}$ of heat stress $\left({ }^{* *} p<0.01,{ }^{\# \#} p<0.01\right)$. The Aspirin + Heat group had lower UA levels than the Heat group at all time points $\left({ }^{\#} p<0.01\right)$. Finally, the Aspirin group had lower UA levels than the Heat group at all time points $\left({ }^{\#} p<0.01\right)$. Lines indicate significant differences between the Aspirin + Heat and Aspirin groups

study, we focus on renal damage in broiler chickens exposed to heat stress, the treatment effect of aspirin under heat stress, and the possible protective role of Hsp47 and Hsp60. Aspirin was reported have a positive effect on chronic heart disease, and chronic kidney disease is a strong independent predictor of cardiovascular disease in human (Evans et al. 2009). After our previous investigation revealed severe heart damage under heat stress and the protective effect of aspirin in broilers, we thought that it was necessary to investigate renal damage and the possible protective effects of aspirin in renal cells too. The animals exposed to heat stress showed increased plasma levels of urea and UA, which was indicative of renal dysfunction. Moreover, histological examination showed swelling of tubular epithelial cells, brush border loss, vacuolar degeneration, necrosis, and decreased renal blood flow; these findings are indicative of renal ischemia and damage. Thus, as observed in cardiac cells in our previous study, renal cells in broilers were also damaged as a result of heat stress.
During heat stress, body temperature increases and the homeostasis of intestinal flora is disrupted, as a result of which the levels of inflammatory factors such as TNF- $\alpha$, IL- 6 , and IL-8 in blood circulation are increased, leading to fever and organ damage. Aspirin has been shown to have antiinflammatory effects and control body temperature. In this study, broilers exposed to heat stress that were not given aspirin presented with higher body temperature and higher urea and UA levels than those that were fed aspirin after exposure to heat stress. Aspirin treatment decreased the urea level in the non-heat-treated group as well, this finding is supported by two studies: (1) when aspirin is present in the body, it increases the Cox-2 level, which can cause a decrease in urea and UA (Wakefield et al. 2012), and (2) aspirin and urea may form a aspirin-urea complex that leads to a decrease in serum urea (Santopadre and Bolton 1967). In addition, examination of the pathological lesions in the treatment and non-treatment groups showed that aspirin could lessen the in vivo damage caused by heat stress in heart cells. Further, the renal samples from the aspirin treatment group were characterized by acute degeneration as opposed to karyopyknosis. Pearson correlation analysis showed that the urea levels were significantly negatively correlated with the Hsp47 and Hsp60 levels. This indicates that Hsp47 and Hsp60 expression may contribute to protecting renal function in broilers. These findings are indicative of the protective role of aspirin in broilers exposed to heat stress. They are also in agreement with previous reports on the protective role of aspirin against heat-induced stress (Murray 1997; Wu et al. 2016; Zhang et al. 2016).

LDH is widely used as an indicator of cellular damage (Bounda et al. 2015; Holm et al. 2012; Tian et al. 2012). In the present study, the serum LDH levels were significantly higher in the heat stress group than in the aspirin treatment group. Aspirin treatment before heat stress seemed to decrease the LDH levels up to $3 \mathrm{~h}$, at which point it showed a sudden increase, but it decreased thereafter to similar levels as in the aspirin only group. These findings indicate that there was severe damage in the heat stress group that was not treated with aspirin, and that aspirin seemed to have a protective effect against oxidative damage induced by heat stress.

Our research group has confirmed through in vivo experiments that Hsp60 can protect the heart and liver from heat stress in broilers and rats (Buriro et al. 2012; Yan et al. 2009). However, there is no research on its protective role in renal failure in heat-stressed broilers. According to our results, in the heat stress group, Hsp60 expression showed a significant increase at 1 to $7 \mathrm{~h}$ after heat stress; this is consistent with the results of a previous study which showed that in human proximal tubule (HPT) cells, after heat stress for $1 \mathrm{~h}$, Hsp60 expression was significantly increased but was decreased from 10 to $24 \mathrm{~h}$ (Somji et al. 2000). This group was also histologically characterized by severe renal damage. The kidney is only second to the heart in mitochondrial abundance 
Fig. 3 Pathological features of the kidney in the different treatment groups. $\mathbf{a}-\mathbf{c}$ In the Aspirin group, no obvious damage to renal tubule epithelial cells can be seen. $\mathbf{d}-\mathbf{f}$ In the Aspirin + Heat group, after $5 \mathrm{~h}$ of heat treatment, the renal tubule epithelial cells showed acute degeneration (upwards arrow); after $24 \mathrm{~h}$ of heat stress, acute degeneration was still the main pathological change, but karyopyknosis (filled triangle) was also observed. $\mathbf{g}-\mathbf{i}$ In the Heat group, severe damage to renal tubule epithelial cells can be observed (upwards arrow), characterized by disappearance of the nucleus and karyopyknosis (filled triangle) (H\&E staining, $\times 400,1$ bar $=20 \mu \mathrm{m}$ )
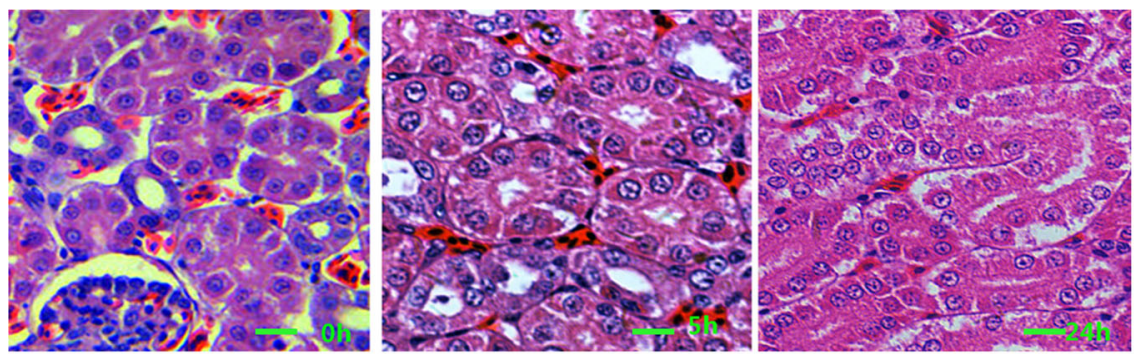

Aspirin
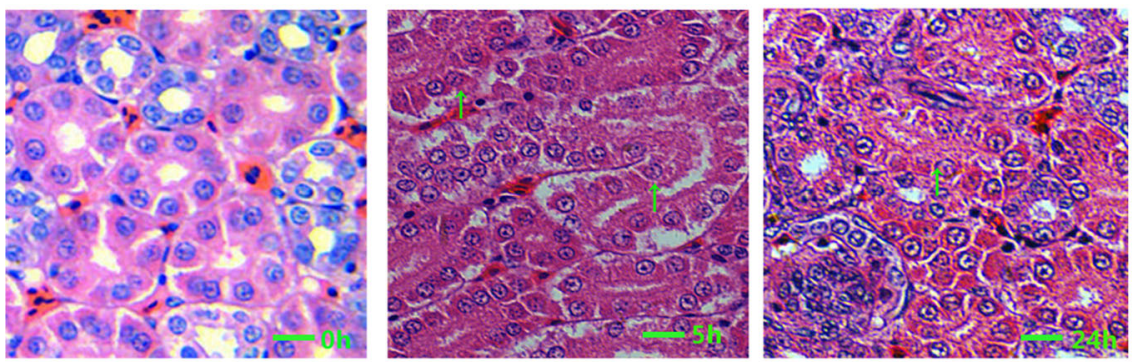

Aspirin

+ Heat
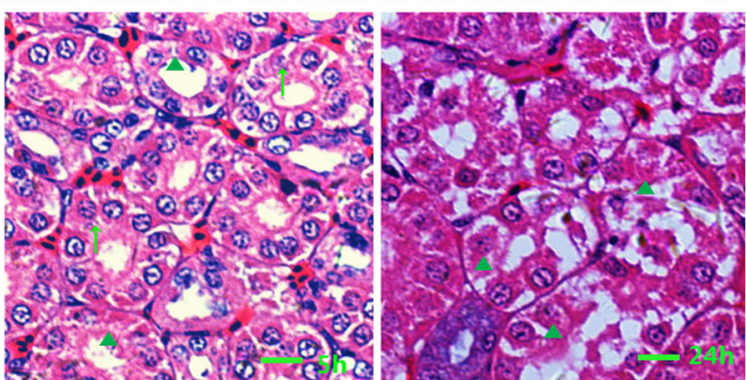

Heat
(Deluca and Engstrom 1961; Mao et al. 2007). Hsp60 is a typical mitochondrial protein in eukaryotes, and is involved in facilitating the correction of misfolded protein back into the correct conformation. Acute heat stress could cause mitochondrial damage; thus, the significant increase in Hsp60 expression might indicate that Hsp60 expression is induced by heat stress to maintain mitochondrial homeostasis (Song et al. 2016). Its expression then decreased from 10 to $24 \mathrm{~h}$ (to $0.47 \mathrm{ng} / \mathrm{mL}$ ) and was accompanied by higher urea and UA levels and corresponding pathological changes (Table 1). Based on the time-dependent changes in Hsp60 expression in the heat-treated and aspirin groups, it seems that aspirin can increase the Hsp60 levels in the unstressed state, Hsp60 expression level in Aspirin-treated chicks before heat shock was about $0.97 \mathrm{ng} / \mathrm{mL}$, no treatment group was $0.75 \mathrm{ng} / \mathrm{mL}$, increased 25\% of Hsp60. After heat stress, Hsp60 levels decreased in Aspirin treatment groups $(0.65 \mathrm{ng} / \mathrm{mL})$ from 1 to $7 \mathrm{~h}$ and then began to increase from 15 to $24 \mathrm{~h}(0.99 \mathrm{ng} / \mathrm{mL})$, and that under heat stress, enough Hsp60 is expressed for maintaining mitochrondrial homeostasis in the early heat stress period. However, after $7 \mathrm{~h}$, the kidney showed damage and induction of Hsp60 expression thereafter. As previous research has shown, Hsp60 is present in both the cytosolic and mitochrondrial areas of cells. Under stress conditions, Hsp60 might bind with mitochondrial membrane proteins to protect mitochondrial membrane potential $(\Delta \Psi \mathrm{m})$. This is supported by the finding that when Hsp60 expression was silenced by siRNA treatment, rat myocardial cells showed a higher apoptosis rate and more ROS production (Song et al. 2016). Further, its expression was similar to that in the aspirin only group at almost all time points. The aspirin-treated groups were also characterized by lighter renal damage. In addition, the findings are consistent with the urea and UA expression profiles. Hsp60 has been proposed to be a regulator of apoptosis in various tissues (Bruschi et al. 1993; Reading et al. 1989; Kuo et al. 2012; Tang et al. 2016); therefore, its mechanism of action may involve apoptotic pathways and also include other positive and negative regulators of cell death and proliferation. In addition, the findings indicate that aspirin may increase expression of Hsp60 within $2 \mathrm{~h}$ in the absence of heat stress.

Hsp47 was originally identified as a heat-inducible glycoprotein in fibroblasts and has been demonstrated to play a major role in the quality control of collagens under heat stress conditions. Reports have indicated that Hsp47 could prevent kidney diseases such as kidney fibrosis, murine antithymocyte serum-induced glomerulonephritis, streptozotocin-induced diabetic nephropathy, human diabetic nephropathy, IgA nephropathy, and hypertensive nephrosclerosis (Razzaque and Taguchi 1999). In the present study, the Hsp47 level decreased from 5 to $24 \mathrm{~h}$ in the heat stress group, but in the Aspirin + Heat group, the Hsp47 level 

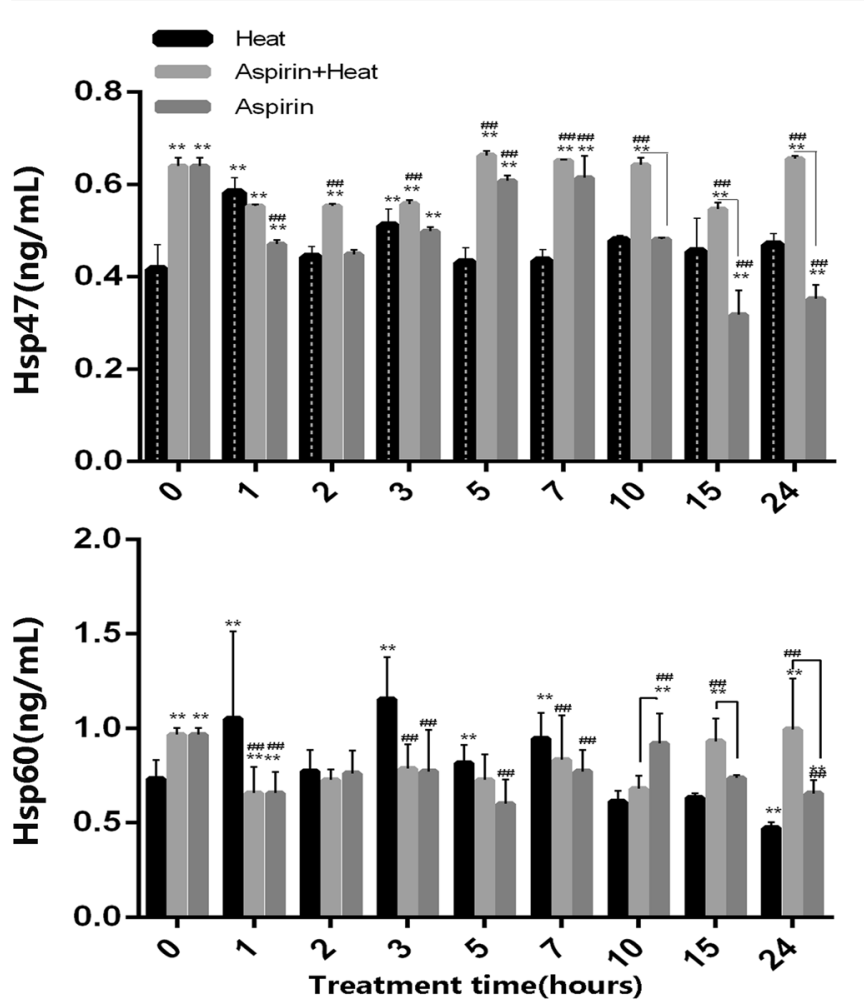

Fig. $4 \mathrm{Hsp} 47$ and Hsp60 expression profiles in the different treatment groups. a Hsp47 was significantly expressed after aspirin pretreatment, and remained at a higher level than in the Heat group until $24 \mathrm{~h}$ of heat stress $\left({ }^{\# \#} p<0.01\right.$ for Aspirin + Heat vs. Heat group). In the heat stress groups, Hsp47 expression increased at 1 and $3 \mathrm{~h}\left({ }^{* *} p<0.01\right.$ for 0 -h heat stress vs. heat stress for different times). The other time points showed similar expression levels to the no heat stress group. The aspirin treatment group showed higher values at 5-7 h, which then decreased until $24 \mathrm{~h}$ $\left({ }^{\# \#} p<0.01\right)$. Lines indicate significant differences between the Aspirin + Heat and Aspirin groups. b Hsp60 expression was pre-induced slightly before heat stress, after which it showed a decreasing trend from 1 to $10 \mathrm{~h}$ of heat stress in the treatment groups, and an increasing trend from 15 to $24 \mathrm{~h}\left({ }^{\# \#} p<0.01\right.$ vs. the heat stress group). In the heat stress group, Hsp60 expression was increased from 1 to $7 \mathrm{~h}$, after which it decreased from 10 to $24 \mathrm{~h}$ of heat stress $\left({ }^{* *} p<0.01\right)$. The aspirin group showed a decreasing trend in Hsp60 expression from 1 to $24 \mathrm{~h}\left({ }^{\# \#} p<0.01\right)$. Lines indicate significant differences between the Aspirin + Heat and Aspirin groups

was induced before heat stress treatment and remained at a higher level than that in the heat stress group. Our previous research has confirmed that after treatment of chicken primary myocardial cells with the Hsp inhibitor BAPTA-AM, more

Table 1 Pearson correlation coefficients (r) among kidney damage indicators of broiler kidney $(n=90)$

Pearson correlation coefficients (r)

\begin{tabular}{lll}
\hline & Hsp47 & Hsp60 \\
\hline UA & $0.377 * *$ & -0.125 \\
Urea & $-0.411 * *$ & $-0.403 * *$
\end{tabular}

*, ** Correlation is significant at the 0.01 level and the 0.05 level, respectively serious damage was observed than in cells exposed to heat stress without BAPTA-AM, even when the cells were pretreated with aspirin. Even though the detailed mechanism of the cardioprotective effect of aspirin remains unclear, research has shown that an appropriate dosage of aspirin can induce the expression of different HSPs in different species (Ebert et al. 2005; Endo et al. 2007; Wischmeyer 2002). Previous studies have suggested that aspirin helps reduce stress injury, a function that is closely related to the induction of HSPs (Ghavami et al. 2002; Jurivich et al. 1992; Wu et al. 2015). This is supported by previous studies which have shown that aspirin can induce the expression of HSPs (Erlejman et al. 2014; Wu et al. 2015, 2016; Zhang et al. 2016). Our Hsp47 expression profile was consistent with heat stress-induced damage. Moreover, Hsp47 was induced at a higher level after aspirin treatment compared to Hsp60. These findings indicate that HSP47 may play a more important renoprotective role against heat stress in broiler chickens than Hsp60, and that Hsp47 could be more easily induced by aspirin than Hsp60. We also investigated other Hsps such as Hsp70 in this study, but their levels did not show any significant differences between the groups (data not shown).

In conclusion, the present findings indicate that heat stress does indeed cause renal damage in broiler chickens and that aspirin plays a protective role against the renal damage induced by heat stress. In addition, the results indicate that HSP60 and HSP47 may be involved in the renoprotective pathways induced by aspirin.

Funding This work was supported by the Fundamental Research Funds for the Central Universities [grant number KJQN201709], the National Natural Science Foundation of China [grant number 31672027], the Jiangsu Natural Science Foundation of China [grant number BK20160732], and the China Postdoctoral Science Foundation [grant number 2016M591860].

\section{Compliance with ethical standards}

Conflict of interest The authors declare that they have no conflicts of interest.

\section{References}

Allakhverdiev SI, Kreslavski VD, Klimov VV, Los DA, Carpentier R, Mohanty P (2008) Heat stress: an overview of molecular responses in photosynthesis. Photosynth Res 98:541-550

Bao E, Sultan KR, Nowak B, Hartung J (2008) Expression and distribution of heat shock proteins in the heart of transported pigs. Cell Stress Chaperones 13:459-466

Barrow MW, Clark KA (1998) Heat-related illnesses. Am Fam Physician 58:749-756

Blum A, Miller H (1998) Role of cytokines in heart failure. Am Heart J 135:181-186

Bounda GA, Zhou W, Wang D, Yu F (2015) Rhein elicits in vitro cytotoxicity in primary human liver HL-7702 cells by inducing 
apoptosis through mitochondria-mediated pathway. Evid Based Complement Altern Med 6-14 2015, 19 pages

Bruschi SA, West KA, Crabb JW, Gupta RS, Stevens JL (1993) Mitochondrial HSP60 (P1 protein) and a HSP70-like protein (mortalin) are major targets for modification during S-(1,1,2,2tetrafluoroethyl)-L-cysteine-induced nephrotoxicity. J Biol Chem 268:23157-23161

Bukau B, Horwich AL (1998) The Hsp70 and Hsp60 chaperone machines. Cell 92:351-366

Buriro R, Lv YJ, Ali I, Tang S, Liu ZJ, Zhang M, Adem A, Hartung J, Bao ED (2012) Temporal variations of Hsp60 and HSF-1 in primary rat myocardial cells in vitro under heat stress. Genet Mol Res GMR 12:3003-3016

Deluca HF, Engstrom GW (1961) Calcium uptake by rat kidney mitochondria. Proc Natl Acad Sci U S A 47:1744-1750

Reading DS, Hallberg RL, Myers AM (1989) Characterization of the yeast HSP60 gene coding for a mitochondrial assembly factor. Nature 337:655-659

Ebert MP et al (2005) Protective role of heat shock protein 27 in gastric mucosal injury. J Pathol 207:177-184. https://doi.org/10.1002/path. 1815

Endo S et al (2007) Geranylgeranylacetone, an inducer of the 70-kDa heat shock protein (HSP70), elicits unfolded protein response and coordinates cellular fate independently of HSP70. Mol Pharmacol 72: 1337-1348. https://doi.org/10.1124/mol.107.039164

Erlejman AG, Lagadari M, Toneatto J, Piwien-Pilipuk G, Galigniana MD (2014) Regulatory role of the 90-kDa-heat-shock protein (Hsp90) and associated factors on gene expression. Biochim Biophys Acta BBA-Gene Regul Mech

Evans M, Fored CM, Bellocco R, Fitzmaurice G, Fryzek JP, Mclaughlin JK, Nyrén O, Elinder CG (2009) Acetaminophen, aspirin and progression of advanced chronic kidney disease. Nephrol Dial Transpl 24:1908-1918

Ghavami A, Nutt MP, Hardy SP (2002) Heat shock protein and high-dose aspirin: effects on random skin flap survival in a rat model. Ann Plast Surg 48:60-67

Hamilton AM, Heikkila JJ (2006) Examination of the stress-induced expression of the collagen binding heat shock protein, hsp47, in Xenopus laevis cultured cells and embryos. Comp Biochem Physiol A Mol Integr Physiol 143:133-141

Holm T, Bruchmann J, Scheynius A, Langel Ü (2012) Cell-penetrating peptides as antifungals towards Malassezia sympodialis. Lett Appl Microbiol 54:39-44

Jurivich DA, Sistonen L, Kroes RA, Morimoto RI (1992) Effect of sodium salicylate on the human heat shock response. Science 255:12431245

Kuo T, Malik Z, Kott KS, Chen L, Knowlton AA (2012) Cardiac myocyte exosome stability and function: role of HSP60. FASEB J 26:1064-1066

Lebret T, Watson R, Molinie V, O'Neill A, Botto H, Fitzpatrick JM (2003) Heat shock proteins -HSP27, HSP60, HSP70, HSP90 - expression in bladder cancer. Cancer 98:88-88

Lin H, Decuypere E, Buyse J (2004) Oxidative stress induced by corticosterone administration in broiler chickens (Gallus gallus domesticus): 2. Short-term effect. Comp Biochem Physiol B Biochem Mol Biol 139:745-751

Mao WP, Ye JL, Guan ZB, Zhao JM, Zhang C, Zhang NN, Jiang P, Tian $\mathrm{T}$ (2007) Cadmium induces apoptosis in human embryonic kidney
(HEK) 293 cells by caspase-dependent and -independent pathways acting on mitochondria. Toxicol in Vitro 21:343-354

Murray WM (1997) Inflammation, aspirin, and the risk of cardiovascular disease. N Engl J Med 337:422-424

Nagata K, Hosokawa N (1996) Regulation and function of collagenspecific molecular chaperone, HSP47. Cell Struct Funct 21:425-430

Razzaque MS, Taguchi T (1999) Localization of HSP47 in cisplatintreated rat kidney: a possible role in tubulointerstitial damage. Clin Exp Nephrol 3:222-228

Santopadre G, Bolton S (1967) Interaction of aspirin with urea in water. J Pharm Pharmacol 19:550-551

Somji, S., Todd, J.H., Sens, M.A., Garrett, S.H., and Sens, D.A. (2000) Expression of heat shock protein 60 in human proximal tubule cells exposed to heat, sodium arsenite and $\mathrm{CdCl}(2)$. Toxicol Lett 115: $127-136$

Song E, Tang S, Xu J, Yin B, Bao E, Hartung J (2016) Lenti-siRNA Hsp60 promote bax in mitochondria and induces apoptosis during heat stress. Biochem Biophys Res Commun 481:125-131

Tang H, Chen Y, Liu X, Wang S, Lv Y, Wu D, Wang Q, Luo M, Deng H (2016) Downregulation of HSP60 disrupts mitochondrial proteostasis to promote tumorigenesis and progression in clear cell renal cell carcinoma. Oncotarget 7:38822-38834

Tang S, Lv Y, Chen H, Adam A, Cheng Y, Hartung J, Bao E (2014) Comparative analysis of $\alpha \mathrm{B}$-crystallin expression in heat-stressed myocardial cells in vivo and in vitro. PLoS One 9:e86937

Tian J, Zeng G, Pang X, Liang M, Zhou J, Fang D, Liu Y, Li D, Jiang L (2012) Identification and immunogenicity of two new HLAA*0201-restricted CD8+ T-cell epitopes on dengue NS1 protein. Int Immunol 24:207-218

Wakefield AP, Ogborn MR, Ibrahim N, Aukema HM (2012) A dietary conjugated linoleic acid treatment that slows renal disease progression alters renal cyclooxygenase-2-derived prostanoids in the Han: SPRD-cy rat. J Nutr Biochem 23:908-914

Wali RK, Wali RK (2010) Aspirin and the prevention of cardiovascular disease in chronic kidney disease. J Am Coll Cardiol 56:966-968

Wischmeyer PE (2002) Glutamine and heat shock protein expression. Nutrition 18:225-228

Wu D, Xv J, Song E, Tang S, Zhang X, Kemper N, Hartung J, Bao E (2015) Acetyl salicylic acid protected against heat stress damage in chicken myocardial cells and may associate with induced Hsp27 expression. Cell Stress Chaperones 20:687-696

Wu D, Zhang M, Xu J, Song E, Lv Y, Tang S, Zhang X, Hartung J, Endong B (2016) In vitro evaluation of aspirin-induced HspB1 against heat stress damage in chicken myocardial cells. Cell Stress Chaperones 21:405-413

Xiao HB, Liu RH, Ling GH, Xiao L, Xia YC, Liu FY, Li J, Liu YH, Chen QK, Lv JL (2012) HSP47 regulates ECM accumulation in renal proximal tubular cells induced by TGF- $\beta 1$ through ERK1/2 and JNK MAPK pathways. Am J Physiol Renal Physiol 303:F757F765

Yan J, Bao E, Yu J (2009) Heat shock protein 60 expression in heart, liver and kidney of broilers exposed to high temperature. Res Vet Sci 86: 533-538

Zhang X, Qian Z, Zhu H, Tang S, Wu D, Zhang M, Kemper N, Hartung J, Bao E (2016) HSP90 gene expression induced by aspirin is associated with damage remission in a chicken myocardial cell culture exposed to heat stress. Br Poult Sci 57:462-473 